\title{
SC1/PRDM4 recruits PRMT5 to control the timing of neural precursor differentiation in developing neural stem cells
}

\section{Alexandra Chittka* and Ursula Grazini}

Wolfson Institute of Biomedical Sciences, University College London, London, UK

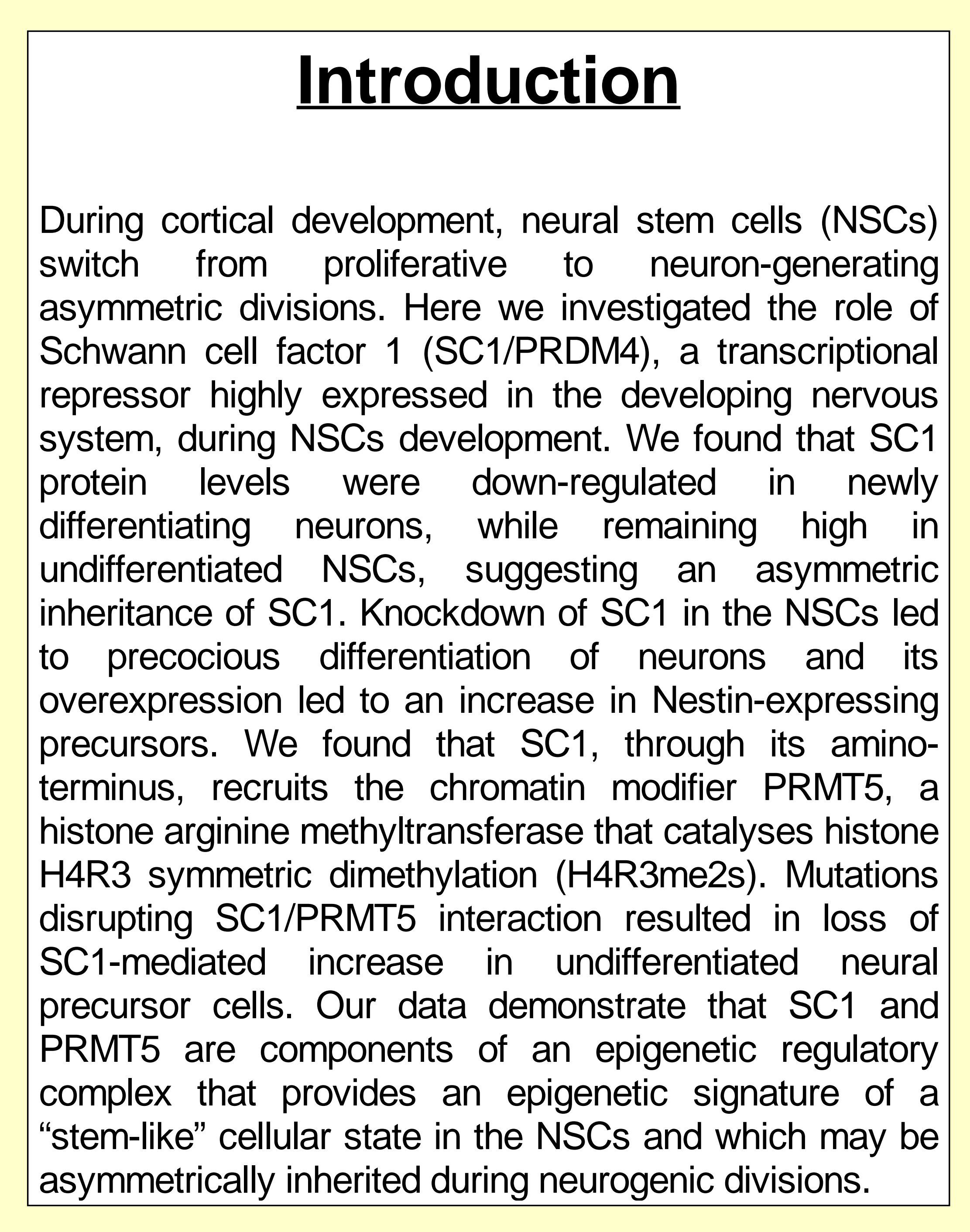

1. SC1/PRDM4 expression in the NSCs is dynamic

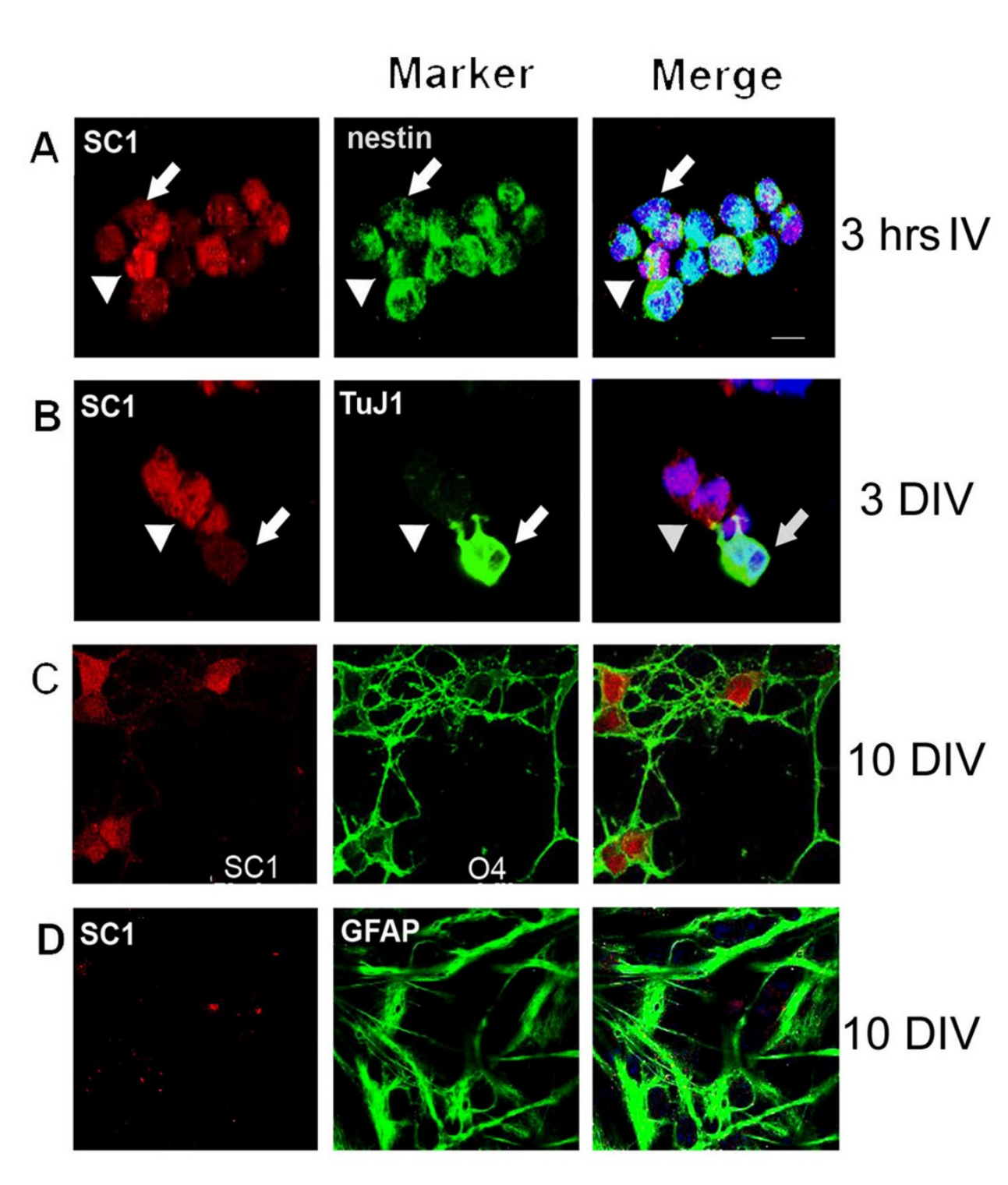

2. SC1/PRDM4 is down-regulated at the onset of neurogenesis and gliogenesis

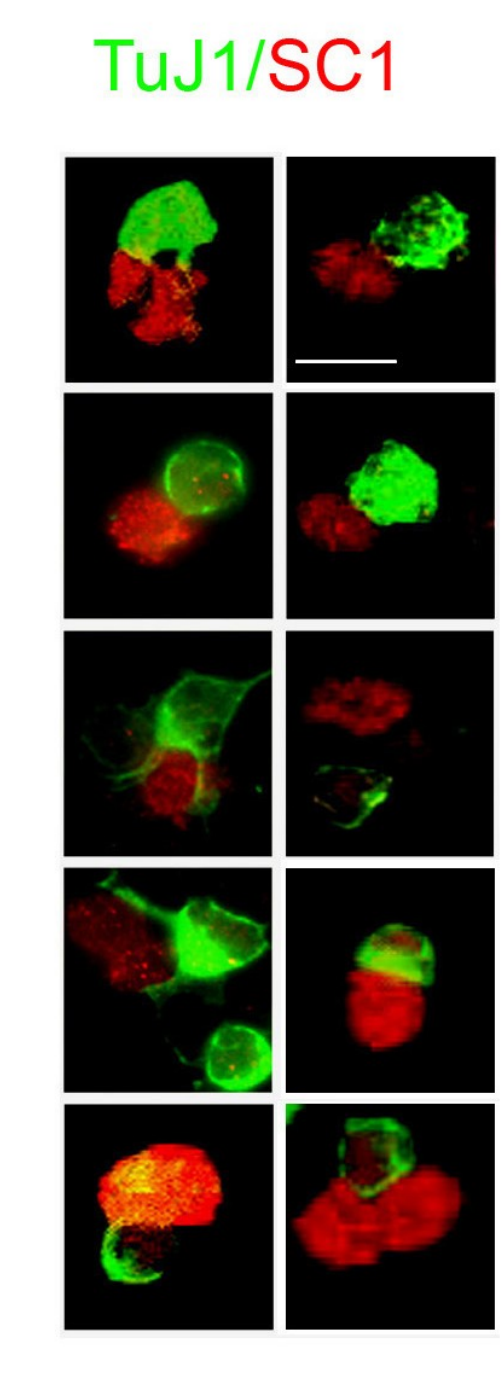

\section{Knockdown of SC1/PRDM4} leads to precocious neurogenesis

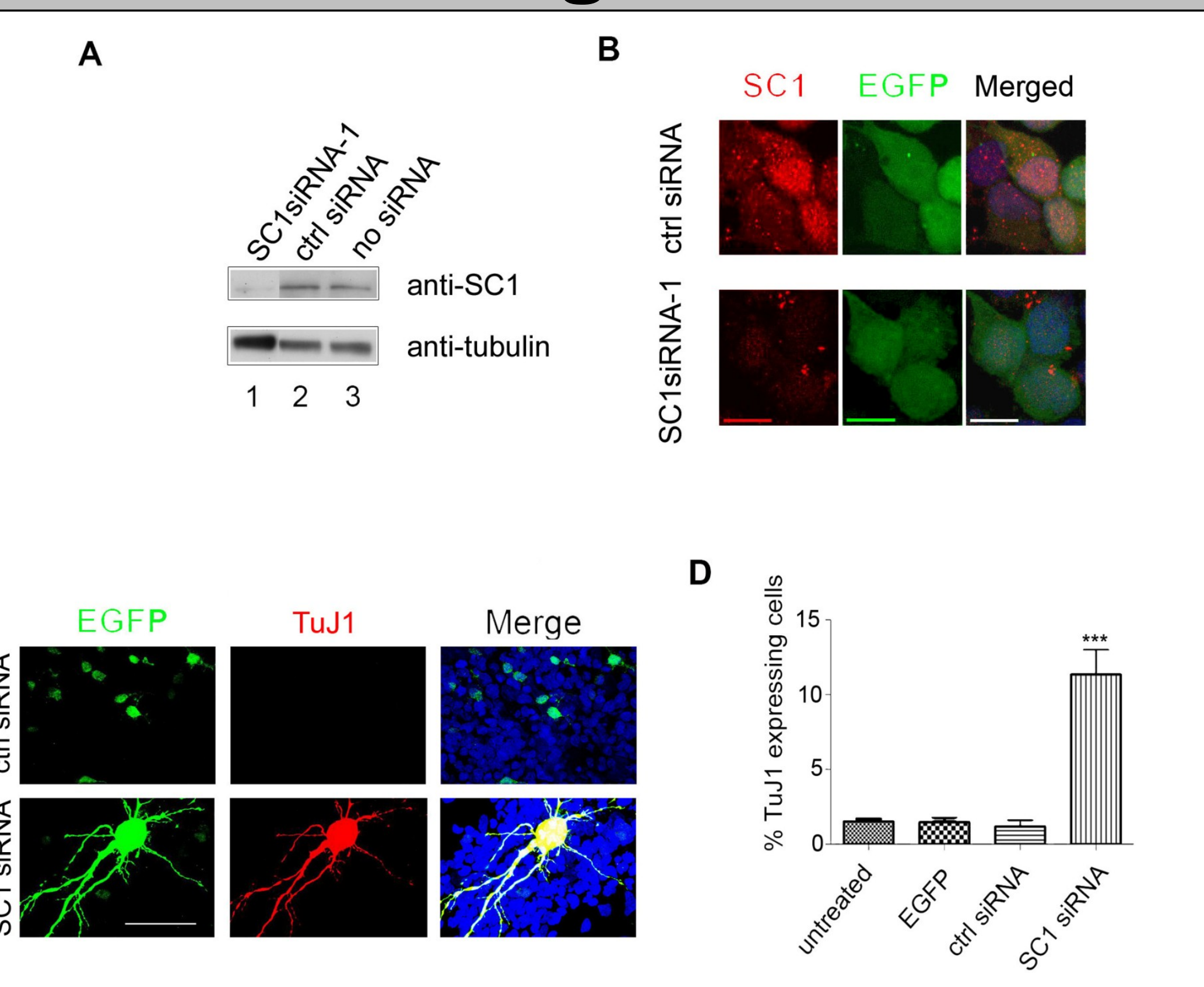

4. SC1/PRDM4 exhibits a histone methyltransferase activity and recruits protein arginine methyltransferase, PRMT5 to mediate symmetric H4R3 dimethylation

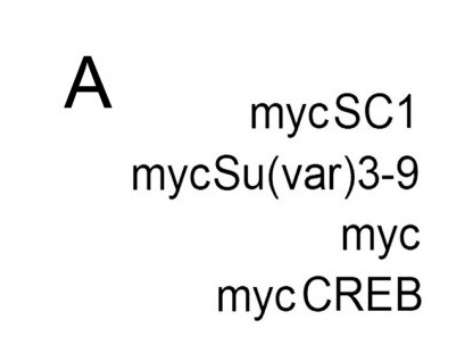

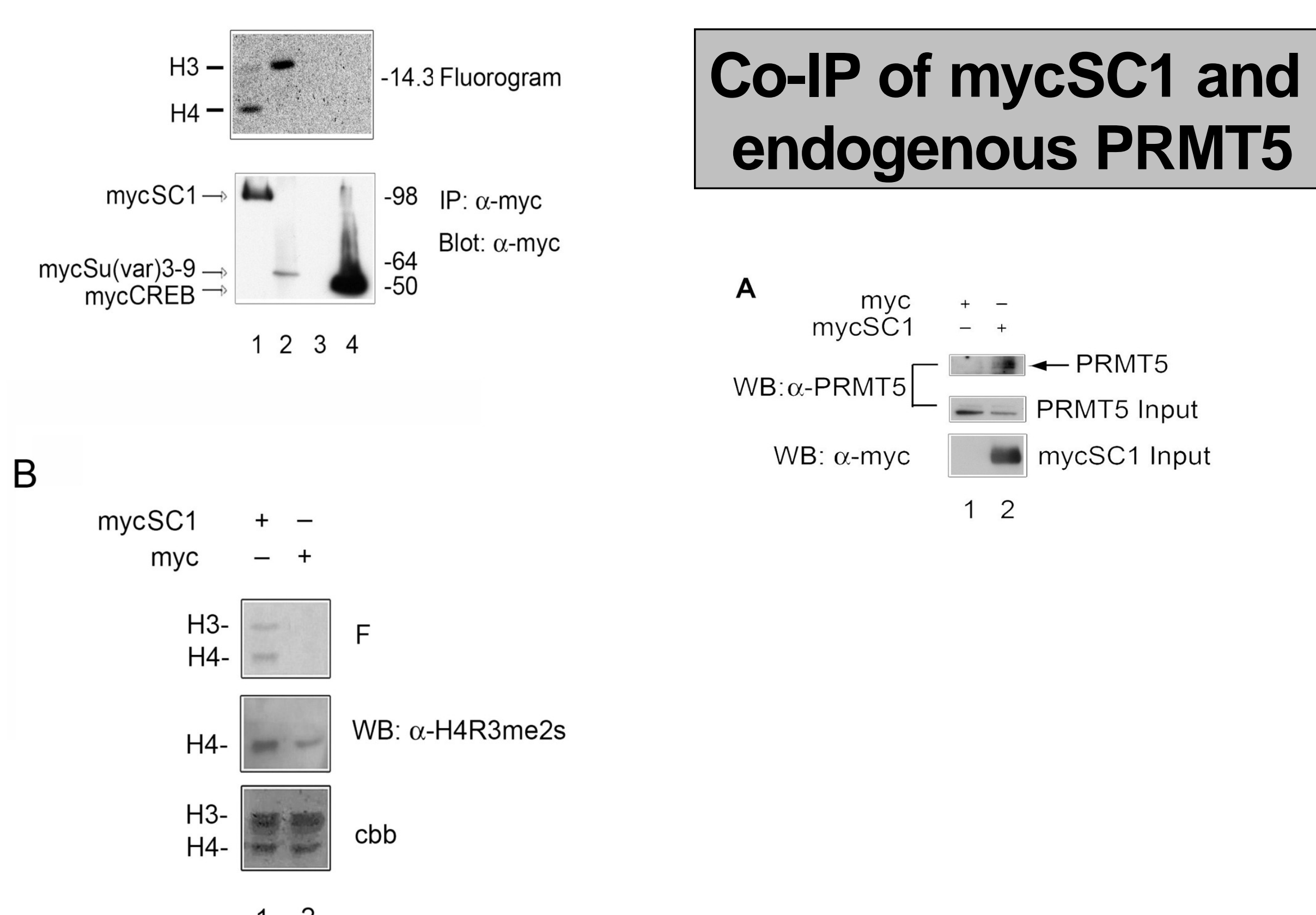

PRMT5 binds to the $\mathrm{NH}$ - and in part to PR/SET domains of SC1/PRDM4

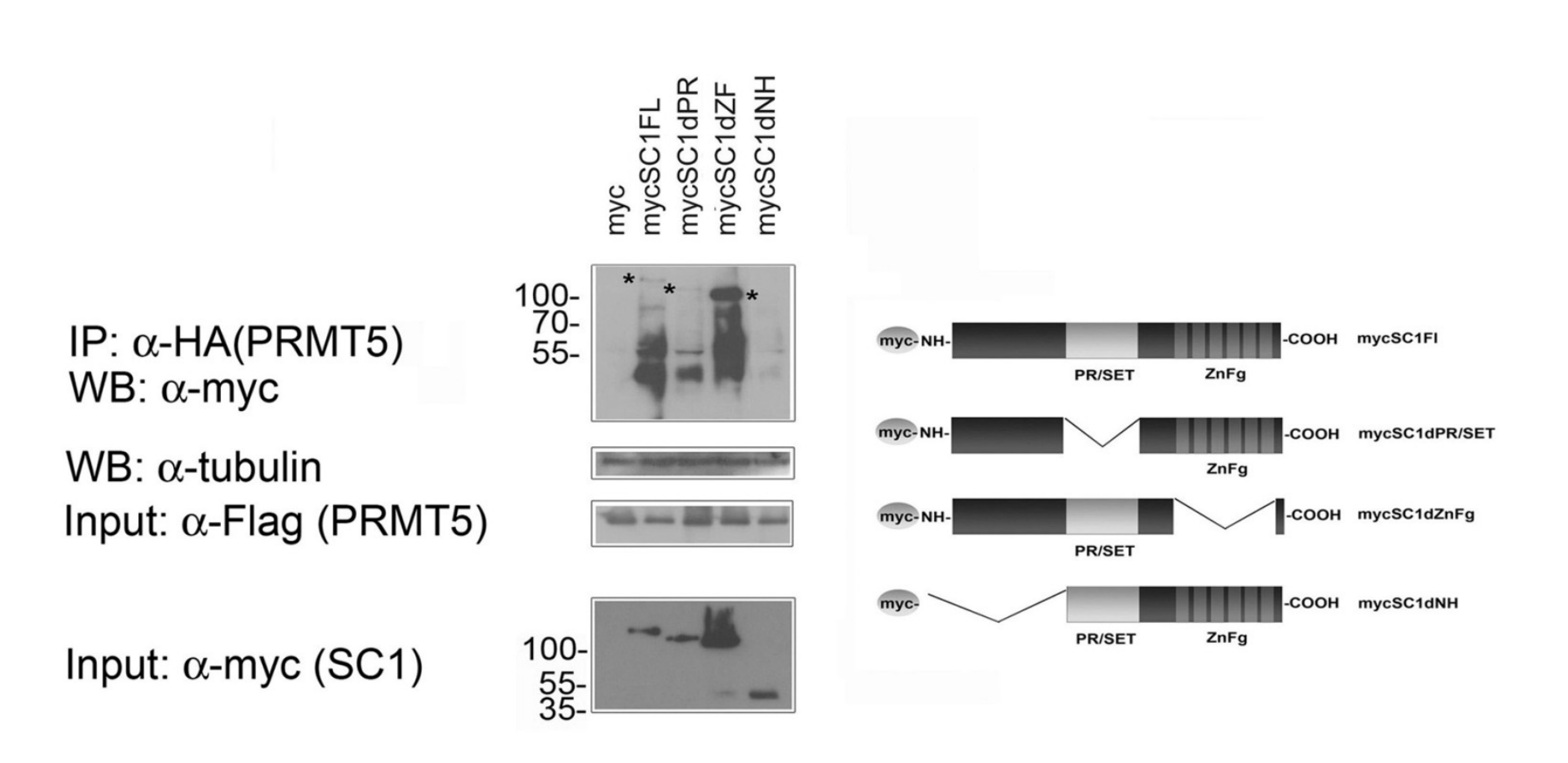

5. PRMT5 is expressed in the neural stem cells

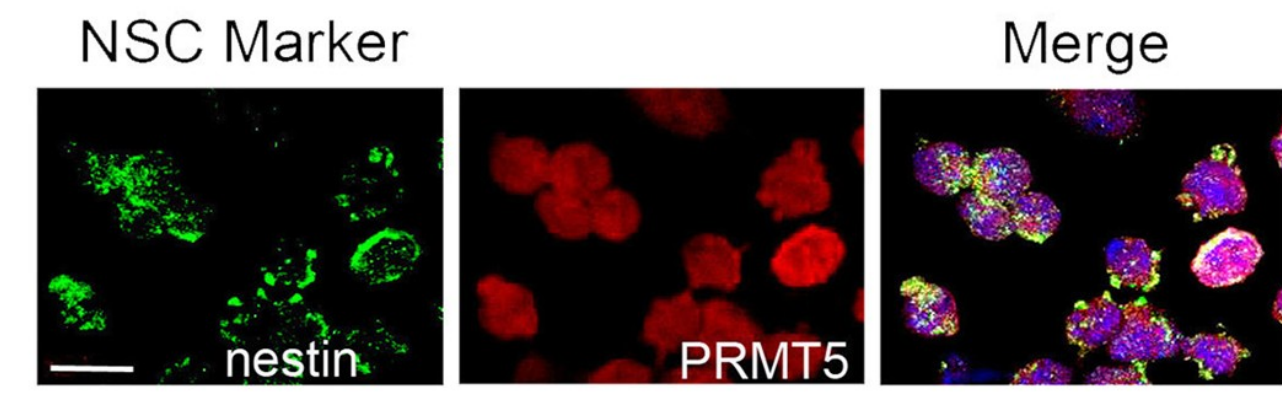

\section{SC1/PRDM4 and PRMT5}

Interaction is necessary

to mediate histone $\mathrm{H} 4$

methylation and

maintain NSCs in an

undifferentiated cellular state

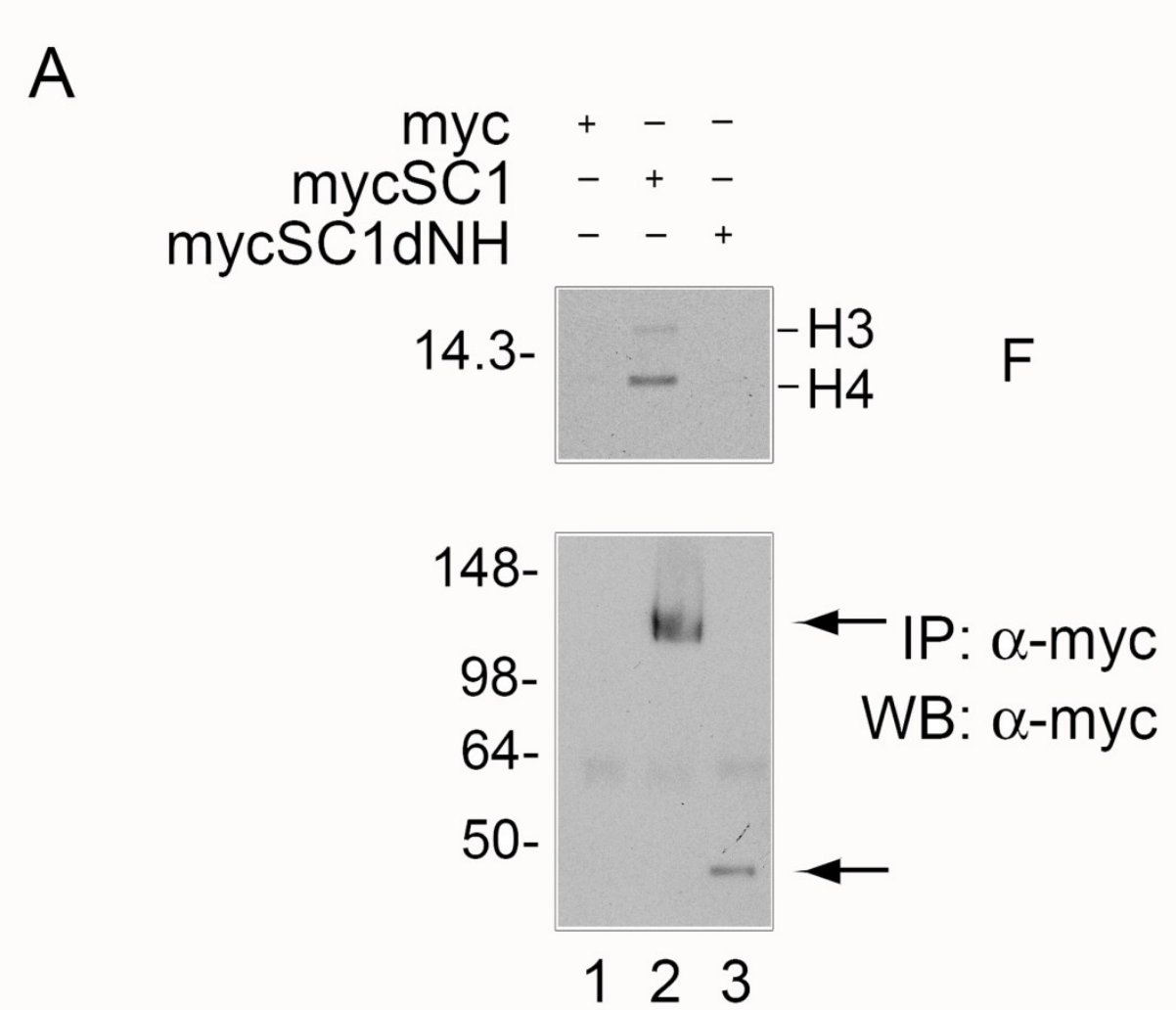

B
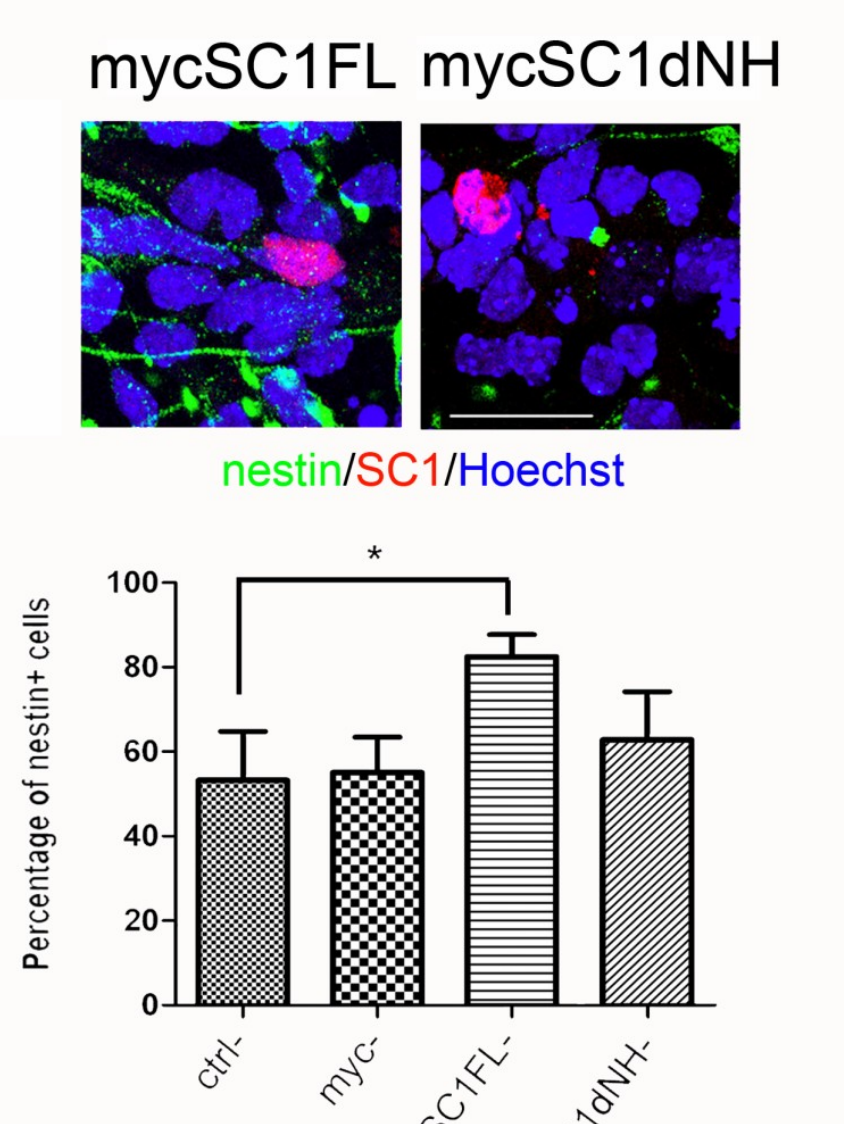

\section{Symmetric H4R3 dimethylation}

is prevalent in the murine cortex at E10.5

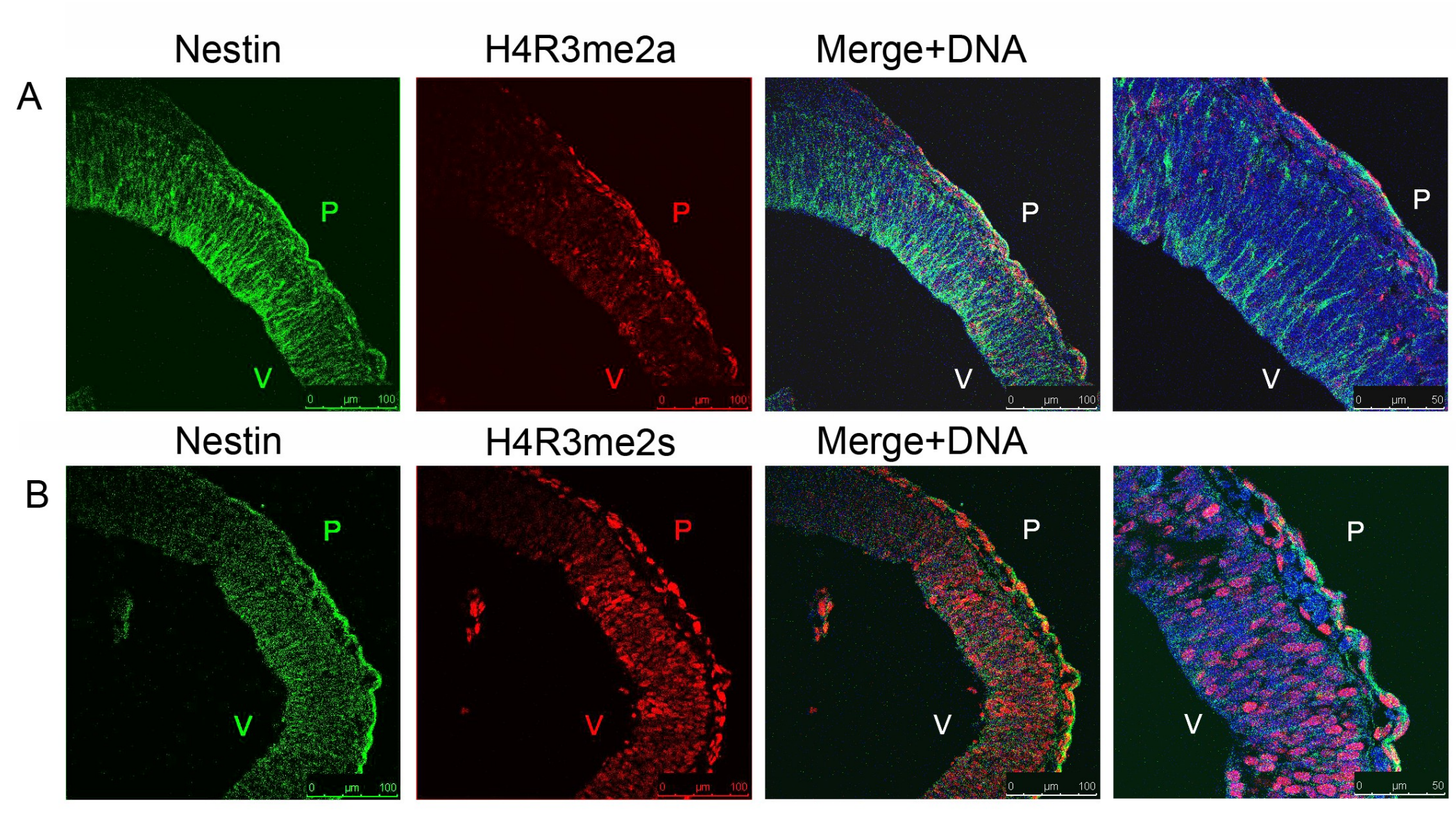

\section{Conclusions}

1) SC1/PRDM4 expression is dynamic in the differentiating neural stem cells

2) SC1/PRDM4 is down-regulated at the onset of neurogenesis and gliogenesis

3) Knockdown of SC1/PRDM4 in neural stem cells leads to precocious neurogenesis

4) $\mathrm{SC1} / \mathrm{PRDM} 4$, through its $\mathrm{NH}$ - and PR/SET domains, recruits PRMT5 to mediate histone H4R3 symmetric dimethylation 5) The complex of SC1/PRDM4 and PRMT5 is necessary to: I) mediate histone $\mathrm{H} 4$ methylation,

m)maintain the undifferentiated cellular state of the NSCs 6) High levels of $\mathrm{H} 4 \mathrm{R} 3$ symmetric dimethylation are found in the developing murine cortex at E10.5 during the proliferative stage of cortical development. 